\title{
STABILITY AND STRONG CONVERGENCE RESULTS FOR RANDOM JUNGCK-KIRK-NOOR ITERATIVE SCHEME
}

\begin{abstract}
The purpose of this study is to introduce a JungckKirk-Noor type random iterative scheme and prove stability and strong convergence of this to establish a general theorem to approximate the unique common random coincidence point for two or more nonself random commuting mappings under general contractive condition in various spaces. Also we give the stability and convergence for random Jungck-Kirk-Ishikawa and random Jungck-Kirk-Mann as a corollaries. The results obtained in this paper improve the corresponding results announced recently.

KEY WORDS: random Jungck-Kirk-Noor iteration, separable Banach spaces, stability results.
\end{abstract}

AMS Mathematics Subject Classification: 47H06, 54H25.

\section{Introduction}

Let $(X, d)$ be a complete metric space, $T: X \rightarrow X$ be a self mapping of $X$. Suppose that $F_{T}=\{x \in X: T(x)=x\}$ is the set of fixed points of $T$. Also the set of all coincidence point of $T, S: Y \rightarrow X$ denoted by $C(S, T)$ such that $C(S, T)=\{z \in Y: S z=T z=p\}$ for an arbitrary set $Y$.

In 2009, Oltainwo [21] introduced the following iterative schemes:

(i) The sequence $\left\{x_{n}\right\}$ defined iteratively by

$$
\left\{\begin{array}{c}
x_{n+1}=\alpha_{n, 0} x_{n}+\sum_{i=1}^{k} \alpha_{n, i} T^{i} y_{n}, \quad \sum_{i=0}^{k} \alpha_{n, i}=1, \\
y_{n}=\alpha_{n, 0} x_{n}+\sum_{j=1}^{s} \beta_{n, j} T^{j} x_{n}, \quad \sum_{j=0}^{k} \beta_{n, j}=1, n=0,1, \ldots
\end{array}\right.
$$

where $k$ and $s$ are fixed integers with $k \geq s, \alpha_{n, i} \geq 0, \alpha_{n, 0} \neq 0, \beta_{n, j} \geq 0$, $\beta_{n, 0} \neq 0$ and $\alpha_{n, i}, \beta_{n, j}$ are sequences in $[0,1]$, is called as Kirk-Ishikawa iterative scheme. 
(ii) The sequence $\left\{x_{n}\right\}$ defined iteratively by

$$
x_{n+1}=\alpha_{n, 0} x_{n}+\sum_{i=1}^{k} \alpha_{n, i} T^{i} x_{n}, \quad \sum_{i=0}^{k} \alpha_{n, i}=1
$$

where $k$ is a fixed integer with $\alpha_{n, i} \geq 0, \alpha_{n, 0} \neq 0$ and $\alpha_{n, i}, \in[0,1]$, is called as Kirk-Mann iterative scheme.

In 2012, Kumar and Chugh [17] studied the convergence of Kirk-Noor iterative scheme with errors as follows: Let $X$ be a Banach space, $K$ be a nonempty closed, convex subset of $X$ and $T_{i}: K \rightarrow K, i=1,2,3$ are self mappings of $K$. Then, the sequence $\left\{x_{n}\right\}_{n=0}^{\infty}$ iteratively by

$$
\left\{\begin{array}{c}
x_{n+1}=\alpha_{n, 0} x_{n}+\sum_{i=1}^{k} \alpha_{n, i} T_{1}^{i} y_{n}+a_{n} u_{n}, \quad \sum_{i=0}^{k} \alpha_{n, i}+a_{n}=1, \\
y_{n}=\beta_{n, 0} x_{n}+\sum_{j=1}^{s} \beta_{n, j} T_{2}^{j} z_{n}+b_{n} v_{n}, \quad \sum_{j=0}^{s} \beta_{n, j}+b_{n}=1, \\
z_{n}=\sum_{l=0}^{t} \gamma_{n, l} T_{3}^{l} x_{n}+c_{n} w_{n}, \quad \sum_{l=0}^{t} \gamma_{n, l}+c_{n}=1, \quad n=0,1,2, \ldots,
\end{array}\right.
$$

where $k, t$ and $s$ are fixed integers with $k \geq s \geq t, \alpha_{n, i} \geq 0, \alpha_{n, 0} \neq 0$, $\beta_{n, j} \geq 0, \beta_{n, 0} \neq 0, \gamma_{n, l} \geq 0, \gamma_{n, 0} \neq 0, \alpha_{n, i}, \beta_{n, j}, \gamma_{n, l}, a_{n}, b_{n}, c_{n} \in[0,1]$ and $u_{n}, v_{n}, w_{n}$ are bounded sequences in $K$.

Many iterative schemes can be obtained from (3) as:

(1) Putting $k=s=t=1$ in (3) we obtain the iterative scheme with errors used by Rashwan et al. [27].

(2) If $t=0$ then Kirk-Noor iteration (3) reduces to Kirk-Ishikawa iteration (2) and taking $t=s=0$ in (3), we have Kirk-Mann iteration (1). For more details see [17].

In 1976, Jungck [14] introduced the following iterative scheme: Let $X$ be a Banach space, $Y$ an arbitrary set and $S, T: Y \rightarrow X$ such that $T(Y) \subseteq S(Y)$, for every $x_{\circ} \in Y$, the sequence $\left\{S x_{n}\right\}_{n=0}^{\infty}$ defined by

$$
S x_{n+1}=T x_{n}, \quad n=0,1, \ldots
$$

This scheme is called Jungck iterative scheme. A lot of authors generalized this scheme to Jungck-Mann, Jungck-Ishikawa, Jungck-Noor and Jungck-SP, these iterations used to approximate the common fixed point for mappings under suitable contractive condition see [1, 4, 10, 11, 12, 15, 19, 20, 22, 24].

The first stability result on $T$-stable mappings was given by Ostrowaski [26] where he proved the stability of the Picard iteration under Banach contraction condition while Osilike et al. [25] improved this result on $(S, T)$ stable as follows: 
Definition 1 ([25]). Let $S, T: Y \rightarrow X$ such that $T(Y) \subseteq S(Y)$, and $z$ a coincidence point of $S$ and $T$, for every $x_{\circ} \in Y$, let the sequence $\left\{S x_{n}\right\}_{n=0}^{\infty}$ generated by the iteration scheme

$$
S x_{n+1}=f\left(T, x_{n}\right), \quad n \geq 0,
$$

converge to $p$. Suppose that $\left\{S y_{n}\right\}_{n=0}^{\infty} \subset X$ be an arbitrary sequence and put $\epsilon_{n}=d\left(S y_{n}, f\left(T, x_{n}\right)\right), n=0,1, \ldots$. Then the iteration scheme (5) will be called $(S, T)$-stable iff $\lim _{n \rightarrow \infty} \epsilon_{n}=0$ implies that $\lim _{n \rightarrow \infty} S y_{n}=p$.

Random fixed point theorems are stochastic generalizations of classical or deterministic fixed point theorems. The theory of random fixed point theorems was initiated in 1950 by Prague school of probabilistic. After the classical results of Bharucha-Reid [3] in 1976, where he gave sufficient conditions for a stochastic analogue of Schouder's fixed point theorem for random operators, the theory of random fixed points received unprecedented attention by several researchers and many interesting results have appeared in the literature see [7, 16, 18, 28]. Špaček [29] and Hanš [8] established stochastic analogue of the Banach fixed point theorem in a separable metric space. Itoh [13] in 1979, generalized and extended Špaček and Hanš's theorem to a multivalued contraction random operators. The development of random fixed point iterations was initiated by Choudhury $[5,6]$, where random Ishikawa iteration scheme was defined and its strong convergence to a random fixed point in Hilbert spaces was discussed.

\section{Preliminaries}

In this paper, we assume that $(\Omega, \Sigma)$ is measurable space consisting of a set $\Omega$ and sigma algebra $\Sigma$ of subset of $\Omega, X$ stands for a separable Banach space or normed space, $C$ is a nonempty closed convex subset of $X$.

A function $T: \Omega \longrightarrow C$ is said to be measurable if $T^{-1}(B \cap C) \in \Sigma$ for each Borel subset $B$ of $X$. A function $T: \Omega \times C \longrightarrow C$ is called a random operator, if $T(., x): \Omega \longrightarrow C$ is measurable for every $x \in C$. A measurable function $f: \Omega \longrightarrow C$ is called a random fixed point for the operator $T: \Omega \times C \longrightarrow C$ if $T(\omega, f(\omega))=f(\omega)$ and it's called random coincidence for two random mappings $S, T: \Omega \times C \longrightarrow C$ if $T(\omega, f(\omega))=$ $S(\omega, f(\omega))$ for all $\omega \in \Omega$. A random operator $T: \Omega \times C \longrightarrow C$ is said to be continuous for any given $\omega \in \Omega, T(\omega,):. \Omega \longrightarrow C$ is continuous. The random operators $T, S: \Omega \times C \longrightarrow C$ are called random commuting if $T(\omega, S(\omega, x))=S(\omega, T(\omega, x))$ for any given $\omega \in \Omega$ and $x \in C$.

The main aim of this paper, is to introduce a new random version of Jungck-Kirk-Noor iterative scheme (3) and study stability and convergence of this to establish a strong convergence to a random coincidence fixed point using general contractive condition for nonself random mappings. 
Now, we introduce random version of iteration schemes as follows:

Let $S, T: \Omega \times Y \rightarrow X$ be two random mappings defined on a nonempty closed convex subset $C$ of a separable Banach space $X$. Let $x_{\circ}: \Omega \rightarrow C$ be an arbitrary measurable mapping for $\omega \in \Omega, n=0,1, \ldots$, with $T(\omega, Y) \subseteq$ $S(\omega, Y)$, then

(i) the sequence $\left\{S\left(\omega, x_{n}(\omega)\right)\right\}_{n=0}^{\infty}$ defined iteratively by:

(6)

$$
\left\{\begin{aligned}
& S\left(\omega, x_{n+1}(\omega)\right)= \alpha_{n, 0} S\left(\omega, x_{n}(\omega)\right) \\
&+\sum_{i=1}^{k} \alpha_{n, i} T^{i}\left(\omega, y_{n}(\omega)\right), \sum_{i=0}^{k} \alpha_{n, i}=1, \\
& S\left(\omega, y_{n}(\omega)\right)= \beta_{n, 0} S\left(\omega, x_{n}(\omega)\right) \\
&+\sum_{j=1}^{s} \beta_{n, j} T^{j}\left(\omega, z_{n}(\omega)\right), \sum_{j=0}^{s} \beta_{n, j}=1, \\
& S\left(\omega, z_{n}(\omega)\right)= \sum_{l=0}^{t} \gamma_{n, l} T^{l}\left(\omega, x_{n}(\omega)\right), \\
& \sum_{l=0}^{t} \gamma_{n, l}=1, \quad n=0,1,2, \ldots,
\end{aligned}\right.
$$

where $k, t$ and $s$ are fixed integers with $k \geq s \geq t, \alpha_{n, i} \geq 0, \alpha_{n, 0} \neq 0$, $\beta_{n, j} \geq 0, \beta_{n, 0} \neq 0, \gamma_{n, l} \geq 0, \gamma_{n, 0} \neq 0$ and $\alpha_{n, i}, \beta_{n, j}, \gamma_{n, l}$ are measurable sequences in $[0,1]$, which is called as Jungck-Kirk-Noor iterative scheme.

(ii) the sequence $\left\{S\left(\omega, x_{n}(\omega)\right)\right\}_{n=0}^{\infty}$ defined iteratively by:

$$
\left\{\begin{aligned}
S\left(\omega, x_{n+1}(\omega)\right)= & \alpha_{n, 0} S\left(\omega, x_{n}(\omega)\right) \\
& +\sum_{i=1}^{k} \alpha_{n, i} T^{i}\left(\omega, y_{n}(\omega)\right), \sum_{i=0}^{k} \alpha_{n, i}=1 \\
S\left(\omega, y_{n}(\omega)\right)= & \beta_{n, 0} S\left(\omega, x_{n}(\omega)\right) \\
& +\sum_{j=1}^{s} \beta_{n, j} T^{j}\left(\omega, x_{n}(\omega)\right), \sum_{j=0}^{s} \beta_{n, j}=1
\end{aligned}\right.
$$

where $k$ and $s$ are fixed integers with $k \geq s, \alpha_{n, i} \geq 0, \alpha_{n, 0} \neq 0, \beta_{n, j} \geq 0$, $\beta_{n, 0} \neq 0$ and $\alpha_{n, i}, \beta_{n, j}$ are measurable sequences in $[0,1]$, which is called as Jungck-Kirk-Ishikawa iterative scheme.

(iii) the sequence $\left\{S\left(\omega, x_{n}(\omega)\right)\right\}_{n=0}^{\infty}$ defined iteratively by:

(8) $S\left(\omega, x_{n+1}(\omega)\right)=\alpha_{n, 0} S\left(\omega, x_{n}(\omega)\right)+\sum_{i=1}^{k} \alpha_{n, i} T^{i}\left(\omega, x_{n}(\omega)\right), \quad \sum_{i=0}^{k} \alpha_{n, i}=1$.

where $k$ is fixed integers, $\alpha_{n, i} \geq 0, \alpha_{n, 0} \neq 0$ and $\alpha_{n, i}$ is measurable sequences in $[0,1]$, which is called as Jungck-Kirk-Mann iterative scheme. 
Remark 1. 1. Putting $t=0$ in (6), we have (7) and taking $t=s=0$ in (6) we get (8).

2. If we take $\Omega$ is a singleton in (6), (7) and (8), we get the nonrandom cases defined in (3), (1) and (2) respectively with $a_{n}=b_{n}=c_{n}=0$ in (3).

The following contractive condition is a stochastic form of (Definition 1, [19]).

Definition 2. For two random mappings $S, T: \Omega \times Y \rightarrow X$ with $T(\omega, Y) \subseteq S(\omega, Y)$ and $C$ is a nonempty closed convex subset of a separable Banach space $X$, there exists real numbers $M \in[0,1], a \in[0,1)$ and a monotone increasing function $\varphi: R^{+} \rightarrow R^{+}$with $\varphi(0)=0$ and for all $x, y \in C$, we have

$$
\begin{aligned}
& \|T(\omega, x)-T(\omega, y)\| \\
& \quad \leq \frac{\varphi(\|S(\omega, x)-T(\omega, x)\|)+a\|S(\omega, x)-S(\omega, y)\|}{1+M\|S(\omega, x)-T(\omega, x)\|} .
\end{aligned}
$$

The following lemmas are useful in the sequel.

Lemma 1 ([2]). If $\delta$ be a real number such that $0 \leq \delta<1$ and $\left\{\epsilon_{n}\right\}_{n=0}^{\infty}$ is a sequence of positive numbers such that $\lim _{n \rightarrow \infty} \epsilon_{n}=0$, then for any sequence of positive numbers $\left\{\rho_{n}\right\}_{n=0}^{\infty}$ satisfying

$$
\rho_{n+1} \leq \delta \rho_{n}+\epsilon_{n}, \quad n=0,1,2, \ldots
$$

we have $\lim _{n \rightarrow \infty} \rho_{n}=0$

Lemma 2. Let $(X,\|\cdot\|)$ be a normed linear space and $S, T$ be random commuting operators on an arbitrary $Y$ with values in $X$ satisfying (9) such that for all $x, y \in Y, \omega \in \Omega$

$$
\left\{\begin{array}{l}
T(\omega, Y) \subseteq S(\omega, Y), \\
\|S(\omega, S(\omega, x))-T(\omega, S(\omega, x))\| \leq\|S(\omega, x)-T(\omega, x)\|, \\
\|S(\omega, S(\omega, x))-S(\omega, S(\omega, y))\| \leq\|S(\omega, x)-S(\omega, y)\| .
\end{array}\right.
$$

Consider $\varphi: R^{+} \rightarrow R^{+}$be a sublinear, monotone increasing function such that $\varphi(0)=0$ and $\varphi(u)=(1-a) u$ for all $a \in[0,1), u \in R^{+}$. Then $\forall k \in N$ and $x, y \in Y$, we have

$$
\left\|T^{k}(\omega, x)-T^{k}(\omega, y)\right\| \leq \frac{\sum_{j=1}^{k}\left(\begin{array}{c}
k \\
j
\end{array}\right) a^{k-j} \varphi^{j}(\|S(\omega, x)-T(\omega, x)\|)}{+a^{k}\|S(\omega, x)-S(\omega, y)\|} .
$$


Proof. In order to prove that $\varphi$ is sublinear see Olatinwo [23, p.15]. The second part of the proof is by mathematical induction (i.e., induction on $k$ ). If $k=1$, then (11) becomes

$$
\begin{gathered}
\|T(\omega, x)-T(\omega, y)\| \leq \frac{\sum_{j=1}^{1}\left(\begin{array}{l}
1 \\
j
\end{array}\right) a^{1-j} \varphi^{j}(\|S(\omega, x)-T(\omega, x)\|)}{1+a\|S(\omega, x)-S(\omega, y)\|} \\
=\frac{\varphi(\|S(\omega, x)-T(\omega, x)\|)+a\|S(\omega, x)-S(\omega, y)\|}{1+M\|S(\omega, x)-T(\omega, x)\|},
\end{gathered}
$$

i.e., (11) reduces to (9) when $k=1$ and hence the result holds.

Assume as an inductive hypothesis that (11) holds for $k=m, m \in N$, i.e.,

$$
\left\|T^{m}(\omega, x)-T^{m}(\omega, y)\right\| \leq \frac{\sum_{j=1}^{m}\left(\begin{array}{c}
m \\
j
\end{array}\right) a^{m-j} \varphi^{j}(\|S(\omega, x)-T(\omega, x)\|)}{+a^{m}\|S(\omega, x)-S(\omega, y)\|} .
$$

We then show that the statement is true for $k=m+1$,

$$
\begin{aligned}
& \left\|T^{m+1}(\omega, x)-T^{m+1}(\omega, y)\right\|=\left\|T^{m}(\omega, T(\omega, x))-T^{m}(\omega, T(\omega, y))\right\| \\
& \leq \frac{\sum_{j=1}^{m}\left(\begin{array}{c}
m \\
j
\end{array}\right) a^{m-j} \varphi^{j}(\|S(\omega, T(\omega, x))-T(\omega, T(\omega, x))\|)}{+a^{m}\|S(\omega, T(\omega, x))-S(\omega, T(\omega, y))\|} \\
& \leq \frac{1+M^{m}\|S(\omega, T(\omega, x))-T(\omega, T(\omega, x))\|}{\sum_{j=1}^{m}\left(\begin{array}{c}
m \\
j
\end{array}\right) a^{m-j} \varphi^{j}(\|T(\omega, S(\omega, x))-T(\omega, T(\omega, x))\|)} \\
& \leq \frac{+a^{m}\|T(\omega, S(\omega, x))-T(\omega, S(\omega, y))\|}{1+M^{m}\|T(\omega, S(\omega, x))-T(\omega, T(\omega, x))\|} \\
& \leq \frac{\sum_{j=1}^{m}\left(\begin{array}{c}
m \\
j
\end{array}\right) a^{m-j} \varphi^{j}\left\{\frac{\varphi(\|S(\omega, S(\omega, x))-T(\omega, S(\omega, x))\|)+a\|S(\omega, S(\omega, x))-S(\omega, T(\omega, x))\|}{1+M\|S(\omega, S(\omega, x))-T(\omega, S(\omega, x))\|}\right\}}{1+M^{m}\left\{\frac{\varphi(\|S(\omega, S(\omega, x))-T(\omega, S(\omega, x))\|)+a\|S(\omega, S(\omega, x))-S(\omega, T(\omega, x))\|}{1+M\|S(\omega, S(\omega, x))-T(\omega, S(\omega, x))\|}\right\}} \\
& +\frac{a^{m}\left\{\frac{\varphi(\|S(\omega, S(\omega, x))-T(\omega, S(\omega, x))\|)+a\|S(\omega, S(\omega, x))-S(\omega, S(\omega, y))\|}{1+M\|S(\omega, S(\omega, x))-T(\omega, S(\omega, x))\|}\right\}}{1+M^{m}\left\{\frac{\varphi(\|S(\omega, S(\omega, x))-T(\omega, S(\omega, x))\|)+a\|S(\omega, S(\omega, x))-S(\omega, T(\omega, x))\|}{1+M\|S(\omega, S(\omega, x))-T(\omega, S(\omega, x))\|}\right\}}
\end{aligned}
$$




$$
\begin{aligned}
& \left.\sum_{j=1}^{m}\left(\begin{array}{c}
m \\
j
\end{array}\right) a^{m-j} \varphi^{j+1}\|S(\omega, x)-T(\omega, x)\|\right) \\
& +\sum_{j=1}^{m}\left(\begin{array}{c}
m \\
j
\end{array}\right) a^{m-j+1} \varphi^{j}\|S(\omega, x)-T(\omega, x)\| \\
& \leq \frac{1+M(\omega, x)-T(\omega, x) \|+M^{m}\{\varphi(\|S(\omega, x)-T(\omega, x)\|)}{1+M(\omega, x)\}} \\
& +a\|S(\omega, x)-T(\omega, x)\|\} \\
& +\frac{a^{m} \varphi(\|S(\omega, x)-T(\omega, x)\|)+a^{m+1}\|S(\omega, x)-S(\omega, y)\|}{1+M\|S(\omega, x)-T(\omega, x)\|+M^{m}\{\varphi(\|S(\omega, x)-T(\omega, x)\|)} \\
& +a\|S(\omega, x)-T(\omega, x)\|\} \\
& \left.\sum_{j=1}^{m}\left(\begin{array}{c}
m \\
j
\end{array}\right) a^{m-j} \varphi^{j+1}\|S(\omega, x)-T(\omega, x)\|\right) \\
& +\sum_{j=1}^{m}\left(\begin{array}{c}
m \\
j
\end{array}\right) a^{m-j+1} \varphi^{j}\|S(\omega, x)-T(\omega, x)\| \\
& =\frac{1+M\|S(\omega, x)-T(\omega, x)\|+M^{m}\|S(\omega, x)-T(\omega, x)\|}{\left.1+{ }^{m}\right)} \\
& +\frac{a^{m} \varphi(\|S(\omega, x)-T(\omega, x)\|)+a^{m+1}\|S(\omega, x)-S(\omega, y)\|}{1+M\|S(\omega, x)-T(\omega, x)\|+M^{m}\|S(\omega, x)-T(\omega, x)\|} \\
& \left.\sum_{j=1}^{m}\left(\begin{array}{c}
m \\
j
\end{array}\right) a^{m-j} \varphi^{j+1}\|S(\omega, x)-T(\omega, x)\|\right) \\
& \leq \frac{+\sum_{j=1}^{m}\left(\begin{array}{c}
m \\
j
\end{array}\right) a^{m-j+1} \varphi^{j}\|S(\omega, x)-T(\omega, x)\|}{1+M^{m+1}\|S(\omega, x)-T(\omega, x)\|} \\
& +\frac{a^{m} \varphi(\|S(\omega, x)-T(\omega, x)\|)+a^{m+1}\|S(\omega, x)-S(\omega, y)\|}{1+M^{m+1}\|S(\omega, x)-T(\omega, x)\|} \\
& \left.\left(\begin{array}{c}
m \\
m
\end{array}\right) \varphi^{m+1}\|S(\omega, x)-T(\omega, x)\|\right)+\left[\left(\begin{array}{c}
m \\
m-1
\end{array}\right)+\left(\begin{array}{c}
m \\
m
\end{array}\right)\right] \\
& =\frac{\times a \varphi^{m}\|S(\omega, x)-T(\omega, x)\|}{1+M^{m+1}\|S(\omega, x)-T(\omega, x)\|} \\
& +\frac{\left[\left(\begin{array}{c}
m \\
m-2
\end{array}\right)+\left(\begin{array}{c}
m \\
m-1
\end{array}\right)\right] a^{2} \varphi^{m-1}\|S(\omega, x)-T(\omega, x)\|+\ldots}{1+M^{m+1}\|S(\omega, x)-T(\omega, x)\|} \\
& {\left[\left(\begin{array}{c}
m \\
2
\end{array}\right)+\left(\begin{array}{c}
m \\
3
\end{array}\right)\right] a^{m-2} \varphi^{3}\|S(\omega, x)-T(\omega, x)\|} \\
& +\frac{+\left[\left(\begin{array}{c}
m \\
1
\end{array}\right)+\left(\begin{array}{c}
m \\
2
\end{array}\right)\right] a^{m-1} \varphi^{2}\|S(\omega, x)-T(\omega, x)\|}{1+M^{m+1}\|S(\omega, x)-T(\omega, x)\|} \\
& +\frac{\left[\left(\begin{array}{c}
m \\
1
\end{array}\right)+\left(\begin{array}{c}
m \\
0
\end{array}\right)\right] a^{m} \varphi\|S(\omega, x)-T(\omega, x)\|+a^{m+1}\|S(\omega, x)-S(\omega, y)\|}{1+M^{m+1}\|S(\omega, x)-T(\omega, x)\|} \\
& \left(\begin{array}{c}
m+1 \\
m+1
\end{array}\right) \varphi^{m+1}\|S(\omega, x)-T(\omega, x)\|+\left(\begin{array}{c}
m+1 \\
m
\end{array}\right) a \varphi^{m}\|S(\omega, x)-T(\omega, x)\| \\
& =\frac{+\left(\begin{array}{c}
m+1 \\
m-1
\end{array}\right) a^{2} \varphi^{m-1}\|S(\omega, x)-T(\omega, x)\|+\ldots}{1+M^{m+1}\|S(\omega, x)-T(\omega, x)\|}
\end{aligned}
$$




$$
+\frac{\begin{array}{c}
\left(\begin{array}{c}
m+1 \\
2
\end{array}\right) a^{m-1} \varphi^{2}\|S(\omega, x)-T(\omega, x)\|+\left(\begin{array}{c}
m+1 \\
1
\end{array}\right) a^{m} \varphi\|S(\omega, x)-T(\omega, x)\| \\
+a^{m+1}\|S(\omega, x)-S(\omega, y)\|
\end{array}}{1+M^{m+1}\|S(\omega, x)-T(\omega, x)\|}
$$

Hence, the proof of Lemma 2 is complete.

\section{Stability results}

Theorem 1. Let $(X,\|\cdot\|)$ be a normed space and $T, S: \Omega \times Y \rightarrow X$ be random commuting operators defined on $Y$ with $T(\omega, Y) \subseteq S(\omega, Y), S(\omega, Y)$ is complete subspace of $X$ satisfying (11) where $\varphi: R^{+} \rightarrow R^{+}$be a sublinear monotone increasing function with $\varphi(0)=0$ and $\alpha_{n, i}, \beta_{n, j}, \gamma_{n, l} \in[0,1]$, $i, j, l=0,1,2, \ldots$. Also $\sum_{i=0}^{k} \alpha_{n, i}=\sum_{j=0}^{s} \beta_{n, j}=\sum_{l=0}^{t} \gamma_{n, l}=1$. Assume that random operators $S, T, S^{i}$ and $T^{i}$ have a random coincidence point say $x(\omega)$. If the sequence $\left\{S\left(\omega, x_{n}(\omega)\right)\right\}_{n=0}^{\infty}$ defined by (6) converges to $p(\omega)$, then the random Jungck-Kirk-Noor iterative scheme is $(S, T)$-stable.

Proof. Let $p(\omega): \Omega \rightarrow C$ be a measurable mapping and $x(\omega): \Omega \rightarrow C$ is random coincidence point for $S, T, S^{i}$ and $T^{i}$ i.e.,

$$
S(\omega, x(\omega))=T(\omega, x(\omega))=S^{i}(\omega, x(\omega))=T^{i}(\omega, x(\omega))=p(\omega) .
$$

Let $\left\{S\left(\omega, z_{n}(\omega)\right)\right\}_{n=0}^{\infty} \subset X$ and

$$
\epsilon_{n}=\left\|S\left(\omega, z_{n+1}(\omega)\right)-\alpha_{n, 0} S\left(\omega, z_{n}(\omega)\right)-\sum_{i=1}^{k} \alpha_{n, i} T^{i}\left(\omega, b_{n}(\omega)\right)\right\|,
$$

where $S\left(\omega, b_{n}(\omega)\right)=\beta_{n, 0} S\left(\omega, z_{n}(\omega)\right)+\sum_{j=1}^{s} \beta_{n, j} T^{j}\left(\omega, m_{n}(\omega)\right)$ and $S\left(\omega, m_{n}(\omega)\right)=$ $\sum_{l=0}^{t} \gamma_{n, l} T^{l}\left(\omega, z_{n}(\omega)\right)$ for every $\omega \in \Omega$.

Let $\lim _{n \rightarrow \infty} \epsilon_{n}=0$, then by Lemma 2 as well as the triangle inequality and (12), we can write

$$
\begin{aligned}
& \left\|S\left(\omega, z_{n+1}(\omega)\right)-p(\omega)\right\| \\
& \leq\left\|S\left(\omega, z_{n+1}(\omega)\right)-\alpha_{n, 0} S\left(\omega, z_{n}(\omega)\right)-\sum_{i=1}^{k} \alpha_{n, i} T^{i}\left(\omega, b_{n}(\omega)\right)\right\| \\
& \quad+\left\|\alpha_{n, 0} S\left(\omega, z_{n}(\omega)\right)+\sum_{i=1}^{k} \alpha_{n, i} T^{i}\left(\omega, b_{n}(\omega)\right)-p(\omega)\right\|
\end{aligned}
$$




$$
\begin{aligned}
= & \epsilon_{n}+\left\|\alpha_{n, 0} S\left(\omega, z_{n}(\omega)\right)+\sum_{i=1}^{k} \alpha_{n, i} T^{i}\left(\omega, b_{n}(\omega)\right)-\sum_{i=0}^{k} \alpha_{n, i} p(\omega)\right\| \\
\leq & \alpha_{n, 0}\left\|S\left(\omega, z_{n}(\omega)\right)-p(\omega)\right\|+\sum_{i=1}^{k} \alpha_{n, i}\left\|T^{i}\left(\omega, b_{n}(\omega)\right)-p(\omega)\right\|+\epsilon_{n} \\
= & \alpha_{n, 0}\left\|S\left(\omega, z_{n}(\omega)\right)-p(\omega)\right\|+\sum_{i=1}^{k} \alpha_{n, i}\left\|T^{i}(\omega, x(\omega))-T^{i}\left(\omega, b_{n}(\omega)\right)\right\|+\epsilon_{n} \\
\leq & \alpha_{n, 0}\left\|S\left(\omega, z_{n}(\omega)\right)-p(\omega)\right\| \\
& +\sum_{i=1}^{k} \alpha_{n, i}\left\{\begin{array}{c}
\sum_{j=1}^{i}\left(\begin{array}{c}
i \\
j
\end{array}\right) a^{i-j} \varphi^{j}(\|S(\omega, x)-T(\omega, x)\|) \\
+a^{i}\left\|S(\omega, x)-S\left(\omega, b_{n}(\omega)\right)\right\| \\
1+M^{i}\|S(\omega, x)-T(\omega, x)\|
\end{array}\right\}+\epsilon_{n} \\
= & \alpha_{n, 0}\left\|S\left(\omega, z_{n}(\omega)\right)-p(\omega)\right\| \\
& +\sum_{i=1}^{k} \alpha_{n, i}\left\{\begin{array}{c}
\sum_{j=1}^{i}\left(\begin{array}{l}
i \\
j
\end{array}\right) a^{i-j} \varphi^{j}(0)+a^{i}\left\|p(\omega)-S\left(\omega, b_{n}(\omega)\right)\right\| \\
\frac{1+M^{i}\|0\|}{j}
\end{array}\right\}+\epsilon_{n},
\end{aligned}
$$

where $\varphi^{j}(0)=0$ hence,

$(13)\left\|S\left(\omega, z_{n+1}(\omega)\right)-p(\omega)\right\| \leq \alpha_{n, 0}\left\|S\left(\omega, z_{n}(\omega)\right)-p(\omega)\right\|$

$$
+\left(\sum_{i=1}^{k} \alpha_{n, i} a^{i}\right)\left\|S\left(\omega, b_{n}(\omega)\right)-p(\omega)\right\|+\epsilon_{n} .
$$

Now, we have the following estimates:

$$
\begin{aligned}
& \left\|S\left(\omega, b_{n}(\omega)\right)-p(\omega)\right\| \\
& \quad=\left\|\beta_{n, 0} S\left(\omega, z_{n}(\omega)\right)+\sum_{j=1}^{s} \beta_{n, j} T^{j}\left(\omega, m_{n}(\omega)\right)-\sum_{j=0}^{s} \beta_{n, j} p(\omega)\right\| \\
& \quad \leq\left\|\beta_{n, 0}\left(S\left(\omega, z_{n}(\omega)\right)-p(\omega)\right)+\sum_{j=1}^{s} \beta_{n, j}\left(T^{j}\left(\omega, m_{n}(\omega)\right)-p(\omega)\right)\right\| \\
& \quad=\beta_{n, 0}\left\|S\left(\omega, z_{n}(\omega)\right)-p(\omega)\right\|+\sum_{j=1}^{s} \beta_{n, j}\left\|T^{j}(\omega, x(\omega))-T^{j}\left(\omega, m_{n}(\omega)\right)\right\| .
\end{aligned}
$$


Applying (11) in above inequality, we have

$$
\left.\begin{array}{l}
\left\|S\left(\omega, b_{n}(\omega)\right)-p(\omega)\right\| \\
\leq \beta_{n, 0}\left\|S\left(\omega, z_{n}(\omega)\right)-p(\omega)\right\| \\
\quad+\sum_{j=1}^{s} \beta_{n, j}\left\{\frac{\left.\begin{array}{c}
\sum_{c=1}^{j}\left(\begin{array}{l}
j \\
c
\end{array}\right) a^{j-c} \varphi^{c}(\|S(\omega, x)-T(\omega, x)\|) \\
+a^{j}\left\|S(\omega, x)-S\left(\omega, m_{n}(\omega)\right)\right\| \\
1+M^{j}\|S(\omega, x)-T(\omega, x)\|
\end{array}\right\}}{=} \beta_{n, 0}\left\|S\left(\omega, z_{n}(\omega)\right)-p(\omega)\right\|\right. \\
\quad+\sum_{j=1}^{s} \beta_{n, j}\left\{\frac{\sum_{c=1}^{j}\left(\begin{array}{l}
j \\
c
\end{array}\right) a^{j-c} \varphi^{c}(0)+a^{j}\left\|S(\omega, x)-S\left(\omega, m_{n}(\omega)\right)\right\|}{1+M^{j}\|(0)\|}\right.
\end{array}\right\},
$$

since $\varphi^{c}(0)=0$, we get

$$
\begin{aligned}
& \left\|S\left(\omega, b_{n}(\omega)\right)-p(\omega)\right\| \\
& \leq \beta_{n, 0}\left\|S\left(\omega, z_{n}(\omega)\right)-p(\omega)\right\|+\sum_{j=1}^{s} \beta_{n, j} a^{j}\left\|p(\omega)-S\left(\omega, m_{n}(\omega)\right)\right\| .
\end{aligned}
$$

Applying (14) in (13), we have

$$
\begin{aligned}
& \left\|S\left(\omega, z_{n+1}(\omega)\right)-p(\omega)\right\| \\
& \quad \leq\left\{\begin{array}{c}
\left(\alpha_{n, 0}+\beta_{n, 0} \sum_{i=1}^{k} \alpha_{n, i} a^{i}\right)\left\|S\left(\omega, z_{n}(\omega)\right)-p(\omega)\right\| \\
\left.+\left(\sum_{i=1}^{k} \alpha_{n, i} a^{i}\right)\left(\sum_{j=1}^{s} \beta_{n, j} a^{j}\right)\right]\left\|S\left(\omega, m_{n}(\omega)\right)-p(\omega)\right\|+\epsilon_{n}
\end{array}\right\} .
\end{aligned}
$$

For estimate $\left\|S\left(\omega, m_{n}(\omega)\right)-p(\omega)\right\|$, then from (6) and (11), we have

$$
\begin{aligned}
\left\|S\left(\omega, m_{n}(\omega)\right)-p(\omega)\right\| & =\left\|\sum_{l=0}^{t} \gamma_{n, l} T^{l}\left(\omega, z_{n}(\omega)\right)-p(\omega)\right\| \\
& =\left\|\sum_{l=0}^{t} \gamma_{n, l} T^{l}\left(\omega, z_{n}(\omega)\right)-\sum_{l=0}^{t} \gamma_{n, l} p(\omega)\right\| \\
& =\sum_{l=0}^{t} \gamma_{n, l}\left\|T^{l}\left(\omega, z_{n}(\omega)\right)-p(\omega)\right\|
\end{aligned}
$$




$$
\begin{aligned}
& =\sum_{l=0}^{t} \gamma_{n, l} \| T^{l}\left(\omega, x(\omega)-T^{l}\left(\omega, z_{n}(\omega)\right) \|\right. \\
& \leq \sum_{l=0}^{t} \gamma_{n, l} a^{l}\left\|S\left(\omega, z_{n}(\omega)\right)-p(\omega)\right\|
\end{aligned}
$$

Using (16) in (15), we get

(17) $\left\|S\left(\omega, z_{n+1}(\omega)\right)-p(\omega)\right\|$

$$
\leq\left\{\begin{array}{c}
\alpha_{n, 0}+\beta_{n, 0} \sum_{i=1}^{k} \alpha_{n, i} a^{i} \\
+\left(\sum_{i=1}^{k} \alpha_{n, i} a^{i}\right)\left(\sum_{j=1}^{s} \beta_{n, j} a^{j}\right)\left(\sum_{l=0}^{t} \gamma_{n, l} a^{l}\right)
\end{array}\right\}\left\|S\left(\omega, z_{n}(\omega)\right)-p(\omega)\right\|+\epsilon_{n} .
$$

Since $a^{i} \in[0,1)$, then

$$
\begin{gathered}
\sum_{i=1}^{k} \alpha_{n, i} a^{i} \leq \sum_{i=1}^{k} \alpha_{n, i}<1-\alpha_{n, 0}, \quad \sum_{j=1}^{s} \beta_{n, j} a^{j} \leq \sum_{j=1}^{s} \beta_{n, j}<1-\beta_{n, 0} \\
\sum_{l=0}^{t} \gamma_{n, l} a^{l} \leq \sum_{l=0}^{t} \gamma_{n, l}=1 .
\end{gathered}
$$

Hence, it follows that

$$
\begin{aligned}
& \alpha_{n, 0}+\beta_{n, 0}\left(\sum_{i=1}^{k} \alpha_{n, i} a^{i}\right)+\left(\sum_{i=1}^{k} \alpha_{n, i} a^{i}\right)\left(\sum_{j=1}^{s} \beta_{n, j} a^{j}\right)\left(\sum_{l=0}^{t} \gamma_{n, l} a^{l}\right) \\
& <\alpha_{n, 0}+\beta_{n, 0}\left(1-\alpha_{n, 0}\right)+\left(1-\alpha_{n, 0}\right)\left(1-\beta_{n, 0}\right)=\delta(\text { say })<1 .
\end{aligned}
$$

Applying (19) and Lemma 1 in (17), we obtain that $\lim _{n \rightarrow \infty}\left\|S\left(\omega, z_{n}(\omega)\right)-p(\omega)\right\|=$ 0 , that is $\lim _{n \rightarrow \infty} S\left(\omega, z_{n}(\omega)\right)=p(\omega)$.

Conversely. consider $\lim _{n \rightarrow \infty} S\left(\omega, z_{n}(\omega)\right)=p(\omega)$, by using both Lemma 2 , the triangle inequality and (17), we have

$$
\begin{aligned}
\epsilon_{n} \leq & \left\|S\left(\omega, z_{n+1}(\omega)\right)-p(\omega)\right\|+\alpha_{n, 0}\left\|S\left(\omega, z_{n}(\omega)\right)-p(\omega)\right\| \\
& +\left(\sum_{i=1}^{k} \alpha_{n, i} a^{i}\right)\left\|S\left(\omega, b_{n}(\omega)\right)-p(\omega)\right\| \\
\leq & \left\|S\left(\omega, z_{n+1}(\omega)\right)-p(\omega)\right\|+\left[\alpha_{n, 0}+\beta_{n, 0} \sum_{i=1}^{k} \alpha_{n, i} a^{i}\right]\left\|S\left(\omega, z_{n}(\omega)\right)-p(\omega)\right\| \\
& +\left(\sum_{i=1}^{k} \alpha_{n, i} a^{i}\right)\left(\sum_{j=1}^{s} \beta_{n, j} a^{j}\right)\left\|S\left(\omega, m_{n}(\omega)\right)-p(\omega)\right\|
\end{aligned}
$$




$$
\begin{aligned}
\leq & \left\|S\left(\omega, z_{n+1}(\omega)\right)-p(\omega)\right\| \\
& +\left\{\begin{array}{c}
\alpha_{n, 0}+\beta_{n, 0} \sum_{i=1}^{k} \alpha_{n, i} a^{i} \\
+\left(\sum_{i=1}^{k} \alpha_{n, i} a^{i}\right)\left(\sum_{j=1}^{s} \beta_{n, j} a^{j}\right)\left(\sum_{l=0}^{t} \gamma_{n, l} a^{l}\right)
\end{array}\right\}\left\|S\left(\omega, z_{n}(\omega)\right)-p(\omega)\right\|,
\end{aligned}
$$

the right hand side of the above inequality tends to 0 as $n \rightarrow \infty$, so $\lim _{n \rightarrow \infty} \epsilon_{n}=0$, the proof is complete.

If we take $t=0$ and $s=t=0$, then from Theorem 1 we get the following corollaries:

Corollary 1. Let $(X,\|\cdot\|)$ be a normed space and $T, S: \Omega \times Y \rightarrow X$ be random commuting operators defined on $Y$ with $T(\omega, Y) \subseteq S(\omega, Y), S(\omega, Y)$ is complete subspace of $X$ satisfying (11) where $\varphi: R^{+} \rightarrow R^{+}$be a sublinear monotone increasing function with $\varphi(0)=0$ and $\alpha_{n, i}, \beta_{n, j} \in[0,1], i=$ $0,1,2, \ldots$. Also $\sum_{i=0}^{k} \alpha_{n, i}=\sum_{j=0}^{s} \beta_{n, j}=1$.

Assume that random operators $S, T, S^{i}$ and $T^{i}$ have a random coincidence point say $x(\omega)$. If the sequence $\left\{S\left(\omega, x_{n}(\omega)\right)\right\}_{n=0}^{\infty}$ defined by (7) converges to $p(\omega)$, then the random Jungck-Kirk-Ishikawa iterative scheme is $(S, T)$-stable.

Corollary 2. Let $(X,\|\cdot\|)$ be a normed space and $T, S: \Omega \times Y \rightarrow X$ be random commuting operators defined on $Y$ with $T(\omega, Y) \subseteq S(\omega, Y), S(\omega, Y)$ is complete subspace of $X$ satisfying (11) where $\varphi: R^{+} \rightarrow R^{+}$be a sublinear monotone increasing function with $\varphi(0)=0$ and $\alpha_{n, i} \in[0,1], i=0,1,2, \ldots$. Also $\sum_{i=0}^{k} \alpha_{n, i}=1$.

Assume that random operators $S, T, S^{i}$ and $T^{i}$ have a random coincidence point say $x(\omega)$. If the sequence $\left\{S\left(\omega, x_{n}(\omega)\right)\right\}_{n=0}^{\infty}$ defined by (8) converges to $p(\omega)$, then the random Jungck-Kirk-Mann iterative scheme is $(S, T)$-stable.

\section{Strong convergence results in separable Banach space}

Theorem 2. Let $X$ be a separable Banach space and $T, S: \Omega \times Y \rightarrow X$ be random commuting operators defined on $Y$ with $T(\omega, Y) \subseteq S(\omega, Y), S(\omega, Y)$ is complete subspace of $X$ and $S^{i}$ is injective satisfying (11) where $\varphi: R^{+} \rightarrow$ $R^{+}$be a sublinear monotone increasing function with $\varphi(0)=0$ and $\alpha_{n, i}, \beta_{n, j}$, $\gamma_{n, l} \in[0,1], i, j, l=0,1,2, \ldots$. Also $\sum_{i=0}^{k} \alpha_{n, i}=\sum_{j=0}^{s} \beta_{n, j}=\sum_{l=0}^{t} \gamma_{n, l}$ 1. Assume 
that random operators $S, T, S^{i}$ and $T^{i}$ have a unique random coincidence point say $x(\omega)$. If the sequence $\left\{S\left(\omega, x_{n}(\omega)\right)\right\}_{n=0}^{\infty}$ defined by (6) converges to $p(\omega)$, then the random Jungck-Kirk-Noor iterative scheme converges strongly to $p(\omega)$.

Proof. Suppose that the sequence $\left\{S\left(\omega, x_{n}(\omega)\right)\right\}_{n=0}^{\infty}$ has a pointwise limit, that is, $\lim _{n \rightarrow \infty} S\left(\omega, x_{n}(\omega)\right)=p(\omega)$ for all $\omega \in \Omega$, since $X$ be a separable Banach space, then the mappings $p(\omega)=S(\omega, f(\omega))$ is measurable mapping for any random operator $S: \Omega \times C \longrightarrow C$ and any measurable mapping $f: \Omega \longrightarrow C[9]$. Therefore the sequence $\left\{S\left(\omega, x_{n}(\omega)\right)\right\}_{n=0}^{\infty}$ constructed by the random Jungck-Ishikawa iteration (7) is a sequence of measurable mappings. Since $p(\omega)$ is measurable and $C$ is convex, then $p: \Omega \rightarrow C$ being limit of measurable mapping sequence is also measurable.

To establish that $S^{i}, T^{i}, S$ and $T$ have a unique coincidence point $x(\omega)$, let $C\left(S^{i}, T^{i}, S, T\right)$ be the set of all coincidence points of $S^{i}, T^{i}, S$ and $T$, suppose that there exists $x_{1}, x_{2} \in C\left(S^{i}, T^{i}, S, T\right)$ such that $S^{i}\left(\omega, x_{1}\right)=$ $T^{i}\left(\omega, x_{1}\right)=S\left(\omega, x_{1}\right)=T\left(\omega, x_{1}\right)=p_{1}(\omega)$ and $S^{i}\left(\omega, x_{2}\right)=T^{i}\left(\omega, x_{2}\right)=$ $S\left(\omega, x_{2}\right)=T\left(\omega, x_{2}\right)=p_{2}(\omega)$.

If $p_{1}(\omega)=p_{2}(\omega)$, therefore $S^{i}\left(\omega, x_{1}\right)=S^{i}\left(\omega, x_{2}\right)$ and $S^{i}$ is injective, it follows that $x_{1}=x_{2}$.

If $p_{1}(\omega) \neq p_{2}(\omega)$, then by using (11), we observe that for every $\omega \in \Omega$ and $i=1,2,3, \ldots$

$$
\begin{aligned}
\left\|p_{1}(\omega)-p_{2}(\omega)\right\| & =\left\|T^{i}\left(\omega, x_{1}\right)-T^{i}\left(\omega, x_{2}\right)\right\| \\
& \leq \frac{\sum_{j=1}^{i}\left(\begin{array}{c}
i \\
j
\end{array}\right) a^{i-j} \varphi^{j}\left(\left\|S\left(\omega, x_{1}\right)-T\left(\omega, x_{1}\right)\right\|\right)}{1+a^{i}\left\|S\left(\omega, x_{1}\right)-S\left(\omega, x_{2}\right)\right\|} \\
\leq & \frac{\left.+\omega, x_{1}\right)-T\left(\omega, x_{1}\right) \|}{1}\left\|a^{i}\right\| S\left(\omega, x_{1}\right)-S\left(\omega, x_{2}\right)\left\|=a^{i}\right\| p_{1}(\omega)-p_{2}(\omega) \|,
\end{aligned}
$$

which leads to $\left(1-a^{i}\right)\left\|p_{1}(\omega)-p_{2}(\omega)\right\| \leq 0$, since $a \in[0,1)$, then $a^{i} \in[0,1)$, from which it follows that $\left\|p_{1}(\omega)-p_{2}(\omega)\right\| \leq 0$ which is a contradiction (the norm is nonnegative). So $\left\|p_{1}(\omega)-p_{2}(\omega)\right\|=0$, implies that $p_{1}(\omega)=$ $p_{2}(\omega)=p(\omega)$, therefore

$$
\begin{aligned}
S^{i}\left(\omega, x_{1}\right) & =T^{i}\left(\omega, x_{1}\right)=S^{i}\left(\omega, x_{2}\right)=T^{i}\left(\omega, x_{2}\right)=S\left(\omega, x_{1}\right) \\
& =T\left(\omega, x_{1}\right)=S\left(\omega, x_{2}\right)=T\left(\omega, x_{2}\right)=p(\omega),
\end{aligned}
$$

it follows that, $S^{i}\left(\omega, x_{1}\right)=S^{i}\left(\omega, x_{2}\right)$ and $S^{i}$ is injective leading to $x_{1}(\omega)=$ $x_{2}(\omega)=x(\omega)$, that is $x(\omega)$ is the unique coincidence point of $S^{i}, T^{i}, S$ and $T$.

We now prove that $\left\{S\left(\omega, x_{n}(\omega)\right)\right\}_{n=0}^{\infty}$ converges strongly to $p(\omega)$. 
It follow from (6), (7), (15) and (16) that for $\omega \in \Omega$,

$$
\begin{aligned}
\| S & \left(\omega, x_{n+1}(\omega)\right)-p(\omega) \| \\
& \leq \alpha_{n, 0}\left\|S\left(\omega, x_{n}(\omega)\right)-p(\omega)\right\|+\sum_{i=1}^{k} \alpha_{n, i} a^{i}\left\|S\left(\omega, y_{n}(\omega)\right)-p(\omega)\right\| \\
& \leq \alpha_{n, 0}\left\|S\left(\omega, x_{n}(\omega)\right)-p(\omega)\right\| \\
+ & \left(\sum_{i=1}^{k} \alpha_{n, i} a^{i}\right)\left(\sum_{j=0}^{s} \beta_{n, j} a^{j}\right)\left\|p(\omega)-S\left(\omega, z_{n}(\omega)\right)\right\| \\
& \leq\left\{\begin{array}{c}
\alpha_{n, 0}+\beta_{n, 0} \sum_{i=1}^{k} \alpha_{n, i} a^{i} \\
+\left(\sum_{i=1}^{k} \alpha_{n, i} a^{i}\right)\left(\sum_{j=1}^{s} \beta_{n, j} a^{j}\right)\left(\sum_{l=0}^{t} \gamma_{n, l} a^{l}\right)
\end{array}\right\}\left\|S\left(\omega, x_{n}(\omega)\right)-p(\omega)\right\| \\
& =\delta\left\|S\left(\omega, x_{n}(\omega)\right)-p(\omega)\right\| \\
\leq & \delta^{n}\left\|S\left(\omega, x_{\circ}(\omega)\right)-p(\omega)\right\| \rightarrow 0 \text { as } n \rightarrow \infty,
\end{aligned}
$$

which mean that $\lim _{n \rightarrow \infty}\left\|S\left(\omega, x_{n+1}(\omega)\right)-p(\omega)\right\|=0$. Therefore random Jungck-Kirk-Noor iteration $\left\{S\left(\omega, x_{n}(\omega)\right)\right\}_{n=0}^{\infty}$ converges strongly to $p(\omega)$. So, we obtain from above inequality $\left\|S\left(\omega, x_{n+1}(\omega)\right)-p(\omega)\right\| \rightarrow 0$ as $n \rightarrow \infty$, i.e. $\left\{S\left(\omega, x_{n}(\omega)\right)\right\}_{n=0}^{\infty}$ converges strongly to $p(\omega)$.

Putting $t=0$ and $t=s=0$, then from Theorem 2, we have the following corollaries:

Corollary 3. Let $X$ be a separable Banach space and $T, S: \Omega \times Y \rightarrow$ $X$ be random commuting mappings defined on $Y$ with $T(\omega, Y) \subseteq S(\omega, Y)$, $S(\omega, Y)$ is complete subspace of $X$ and $S^{i}$ is injective satisfying (11) where $\varphi: R^{+} \rightarrow R^{+}$be a sublinear monotone increasing function with $\varphi(0)=0$ and $\alpha_{n, i}, \beta_{n, j} \in[0,1], i=0,1,2, \ldots$ Also $\sum_{i=0}^{k} \alpha_{n, i}=\sum_{j=0}^{s} \beta_{n, j}=1$. Suppose that random operators $S, T, S^{i}$ and $T^{i}$ have a unique random coincidence point say $x(\omega)$. If the sequence $\left\{S\left(\omega, x_{n}(\omega)\right)\right\}_{n=0}^{\infty}$ defined by (7) converges to $p(\omega)$, then the random Jungck-Kirk-Ishikawa iterative scheme convergence strongly to $p(\omega)$.

Corollary 4. Let $X$ be a separable Banach space and $T, S: \Omega \times Y \rightarrow$ $X$ be random commuting mappings defined on $Y$ with $T(\omega, Y) \subseteq S(\omega, Y)$, $S(\omega, Y)$ is complete subspace of $X$ and $S^{i}$ is injective satisfying (11) where $\varphi: R^{+} \rightarrow R^{+}$be a sublinear monotone increasing function with $\varphi(0)=0$ and $\alpha_{n, i} \in[0,1], i=0,1,2, \ldots$ Also $\sum_{i=0}^{k} \alpha_{n, i}=1$. Suppose that random operators $S, T, S^{i}$ and $T^{i}$ have a unique random coincidence point say $x(\omega)$. 
If the sequence $\left\{S\left(\omega, x_{n}(\omega)\right)\right\}_{n=0}^{\infty}$ defined by (8) converges to $p(\omega)$, then the random Jungck-Kirk-Mann iterative scheme convergence strongly to $p(\omega)$.

Acknowledgement: The authors would like to thank the chief editor and the referee for his/her careful reading and making a useful comments which improved the presentation and the readability of the paper.

\section{References}

[1] Alotaibi A., Kumar V., Hussain N., Convergence comparison and stability of Jungck- Krik-type algorithms for common fixed point problems, Fixed Point Theory Appl., 173(2013), 1-30.

[2] Berinde V., On the convergence of the Ishikawa iteration in the class of quasi-contractive operators, Acta Math. Univ. Comenianae, 73(2004), $119-126$.

[3] Bharucha-Reid A.T., Fixed point theorems in probabilistic analysis, Bull. Amer. Math. Soc., 82(1976), 641-657.

[4] Bosede, Olufemi A., On the stability of Jungck-Mann, Jungck-Krasnoselskij and Jungck iteration process in arbitrary Banach spaces, Acta Univ. Palacki. Olomuc. Fac. Rer. Nat. Mathematica, 50(2011), 17-22.

[5] Choudhury B.S., Convergence of a random iteration scheme to a random fixed point, J. Appl. Math. Stochastic Anal., 8(1995), 139-142.

[6] Choudhury B.S., A random fixed point iteration for three random operators on uniformly convex Banach spaces, Anal. Theory Appl., 19(2003), 99-107.

[7] Chugh R., Kumar V., Narwal S., Some strong convergence results of random iterative algorithms with errors in Banach spaces, Commun. Korean Math. Soc., 31(1)(2016), 147-161.

[8] Hanš O., Reduzierende zufăllige transformationen, Czechoslov. Math. J., 7 (1957), 154-158.

[9] Himmelberg C.J., Measurable relations, Fund. Math., 87(1975), 53-71.

[10] Hussain N., Chugh R., Kumar V., RafiQ A., On the rate of convergence of Kirk type iterative schemes, J. Appl. Math., Article ID 526503(2012), 1-22.

[11] Hussain N., Kumar V., Chugh R., Malik P., Jungck-type implicit iterative algorithms with numerical examples, Filomat, 31(2017), 2303-2320.

[12] IoAna, Stability of Jungck-type iterative procedure for some contractive type mappings via implicit relations, Miskolc Math. Notes, 13(2012), 555-567.

[13] Ітон S., Random fixed-point theorems with an application to random differential equations in Banach spaces, J. Math. Anal. Appl., 67(1979), 261-273.

[14] Jungck G., Commuting mappings and fixed points, Amer. Math. Manthly, 83(1976), 261-263.

[15] Khan A.R., Kumar V., Hussain N., Analytical and numerical treatment of Jungck type iterative schemes, Appl. Math. Comput., 231(2014), 521-535.

[16] Khan A.R., Kumar V., Narwal S., Chugh R., Random iterative algorithms and almost sure stability in Banach Spaces, Filomat, 31(2017).

[17] Kumar V., Chugh R., Strong convergence of hybrid fixed point iterative algorithms of Kirk-Noor type with errors in an arbitrary Banach space, Int. J. Pure Appl. Math., 80(2012), 161-171. 
[18] Lin T.C., Random approximation and random fixed point theorems for non self mappings, Proc. Amer. Math. Soc., 103(1988), 1129-1135.

[19] Olatinwo M.O., A Generalization of some convergence results using the Jungck-Noor three step iteration process in arbitrary Banach space, Fasciculi Math., 40(2008), 37-43.

[20] Olatinwo M.O., Some stability and strong convergence results for the Jungck-Ishikawa iteration process, Creative Math. Informatics, 17(2008), $33-42$.

[21] Olatinwo M.O., Some stability results for two hybrid fixed point iterative algorithms in normed linear space, Mathematique Vesnik, 61(2009), 247-256.

[22] Olatinwo M.O., Convergence results for Jungck-type iterative process in convex metric spaces, Acta Univ. Palacki Olomue, Fac. Rer. Nat. Math., 51(2012), 79-87.

[23] Olatinwo M.O., Stability results for Jungck-kirk-Mann and Jungck-kirk hybrid iterative algorithms, Anal. Theory Appl., 29(2013), 12-20.

[24] Olatinwo M.O., Imoru C.O., Some convergence result for the JungckMann and Jungck-Ishikawa iteration process in the class of generalized Zamfirescu operators, Acta Math. Univ. Comenianae, 77(2008), 299-304.

[25] Osilike M.O., Udomene A., Short proofs of stability results for fixed point iteration procedures for a class of contractive-type mappings, Indian J. Pure Appl. Math., 30(12)(1999), 1229-1234.

[26] Ostrowski A.M., The round-off stability of iterations, Z. Angew. Math. Mech., 47(1967), 77-81.

[27] Rashwan R.A., Rafiq A., Hakim A., On the convergence of three-steps iteration process with errors in the class of quasi-contractive, Paki. Acad. Sci., 46(2009), 41-46.

[28] Rashwan R.A., Hammad H.A., Okeke G.A., Convergence and almost sure $(S, T)$-stability for random iterative schemes, Int. J. Advances in Math., 2016(2016), 1-16.

[29] ŠPAČEK A., Zufăllige Gleichungen, Czechoslovak, Math. J., 5(1955), 462-466.

\author{
R.A. RASHWAN \\ Department of Mathematics \\ FACULTY OF SCIENCE \\ Assuit University \\ Assuit 71516, EGYPT \\ e-mail: rr_rashwan54@yahoo.com \\ H.A. HAMMAD \\ Department of Mathematics \\ FACULTY OF SCIENCE \\ SOHAG UNIVERSITY \\ SoHAG 82524, EGYPT \\ e-mail: h.elmagd89@yahoo.com
}

Received on 30.08.2016 and, in revised form, on 16.05.2017. 